\title{
Refinement of Multiparameters Overrelaxation (RMPOR) Method
}

\author{
Gashaye Dessalew $(\mathbb{D}$, Tesfaye Kebede $(\mathbb{D}$, Gurju Awgichew $\mathbb{D}$, and Assaye Walelign $(\mathbb{D}$ \\ Department of Mathematics, Bahir Dar University, Bahir Dar, Ethiopia \\ Correspondence should be addressed to Tesfaye Kebede; tk_ke@yahoo.com
}

Received 31 May 2021; Accepted 12 August 2021; Published 23 August 2021

Academic Editor: Jia-Bao Liu

Copyright ( 92021 Gashaye Dessalew et al. This is an open access article distributed under the Creative Commons Attribution License, which permits unrestricted use, distribution, and reproduction in any medium, provided the original work is properly cited.

\begin{abstract}
In this paper, we present refinement of multiparameters overrelaxation (RMPOR) method which is used to solve the linear system of equations. We investigate its convergence properties for different matrices such as strictly diagonally dominant matrix, symmetric positive definite matrix, and M-matrix. The proposed method minimizes the number of iterations as compared with the multiparameter overrelaxation method. Its spectral radius is also minimum. To show the efficiency of the proposed method, we prove some theorems and take some numerical examples.
\end{abstract}

\section{Introduction}

Large and sparse linear systems of the form

$$
A x=b \text {, }
$$

can be solved using iterative methods. One of the techniques of obtaining iterative methods is splitting the coefficient matrix. $A=M-N$ and $A=D-\sum_{i=1}^{k} E_{i}-F$ are some of the splitting of the matrix $A$. Using either of splittings, we can derive iterative methods which can solve the system of linear equation (1).

Recently, many researchers used the splitting $A=D-\sum_{i=1}^{k} E_{i}-F$, where $D$ is diagonal matrix of $A$, $\sum_{i=1}^{k} E_{i}$ is strictly lower triangular matrix of $D-A$, and $F$ is strictly upper triangular matrix of $D-A$, to get the new iterative methods. Song and Dai [1] introduced multiparameters overrelaxation (MPOR) method, whose special cases involve many classical iterative methods. The convergence conditions with $\mathrm{A}$ being a Hermitian matrix, an L-matrix, an M-matrix, an H-matrix, and a strictly diagonally dominant matrix are derived. Kuang and Ji [2] presented a two-parameter iterative method called TOR method, which is effective to give the numerical solution of partial differential equations. Wang extended the TOR method to the GTOR method and improved some results of Ju, Wang, and Zeng. In [3], O'Leary and White proposed multisplitting methods which are based on several splittings of the matrix A. More precisely, a multisplitting of A is defined as a collection of triples $\left(M_{k}, N_{k}, E_{k}\right)$, $k=1,2, \ldots, K$, such that for all $k, M_{k}, N_{k}$, and $E_{k}$ are $n \times n$ matrices, each $M_{k}$ is nonsingular, $A=M_{k}-N_{k}$, and $E_{k}$ is a diagonal matrix with nonnegative entries satisfying $\sum_{i=1}^{k} E_{k}=I$. The corresponding multisplitting method to solve (1) is given by the iteration $x^{m+1}=\sum_{i=1}^{k} E_{k} y^{m, k}$, $m=0,1,2, \ldots$, where $M_{k} y^{m, k}=N_{k} x^{m}+b, k=1,2, \ldots, K$. Similarly, the convergence of the parallel multisplitting TOR method is studied for M-matrix in [4]. Chang studied the convergence of the parallel multisplitting TOR method for $\mathrm{H}$-matrices, but Zhang et al. [5] found some gaps in the proof of theorem.

There are other many techniques to get new iterative methods such as extrapolation, overrelaxation, and acceleration. Up to now, a lot of first-order stationary iterative methods are proposed. They include the well-known methods as Jacobi, JOR, Gauss-Seidel, SOR, AOR, GAOR, TOR, and so on [1].

Partial differential equations (PDEs) can be solved using finite difference and finite element methods. These two methods have deficiencies in accuracy and code implementation but there is novel efficient matrix approach for solving the second-order linear matrix partial differential equations (MPDEs) under given initial conditions which are 
proposed by Tohidi and Khorsand Zak [6]. Similarly, Asgari et al. [7] introduced an extended block Golub-Kahan algorithm for large algebraic and differential matrix Riccati equations by providing new theoretical analysis of the method and the norm of the residual.

The remainder of this paper is organized as follows. In Section 2, we first review some preliminaries which are used in our basic idea. In Section 3, the main result of this paper is presented. Convergence analysis of the proposed scheme is provided, and numerical examples are considered in this section. In Section 4, we provide a brief conclusion from our main results.

\section{Preliminaries}

The solution of equation (1) can be obtained using the following methods:

(i) SOR: $x^{(n+1)}=(D-\omega E)^{-1}((1-\omega) D+\omega F) x^{(n)}+\omega$ $(D-\omega E)^{-1} b$

(ii) AOR: $\quad x^{(n+1)}=(D-\widehat{\gamma} E)^{-1}((1-\widehat{\omega}) D+(\widehat{\omega}-\widehat{\gamma}) E+$ $\widehat{\omega} F) x^{(n)}+\widehat{\omega}(D-\widehat{\gamma} E)^{-1} b$

(iii) TOR: $\quad x^{(n+1)}=\left(D-a_{1} E_{1}-a_{2} E_{2}\right)^{-1}((1-\omega) D+$ $\left.\left(\omega-a_{1}\right) E_{1}+\left(\omega-a_{2}\right) E_{2}+\omega F\right) x^{(n)}+\omega\left(D-a_{1} E_{1}-\right.$ $\left.a_{2} E_{2}\right)^{-1} b$

In [2], the lower part of $A$ is split into two parts: $E_{1}$ and $E_{2}$. Also, $E_{1}$ and $E_{2}$ are multiplied different factors, and this a new scheme was introduced.

(iv) MPOR: $x^{(n+1)}=\left(D-\sum_{i=1}^{k} a_{i} E_{i}\right)^{-1}\left[(1-\omega) D+\sum_{i=1}^{k}\right.$ $\left.\left(\omega-a_{i}\right) E_{i}+\omega F\right] x^{(n)}+\omega\left(D-\sum_{i=1}^{k} a_{i} E_{i}\right)^{-1} b$

Definition 1. The splitting $A=M-N$ is called

(1) Regular if $M^{-1} \geq O$ and $N \geq O$

(2) Weak regular if $M^{-1} \geq O$ and $M^{-1} N \geq O$

Theorem 1. Let $A=M-N$ be a regular splitting of a matrix $A$. Then, $\rho\left(M^{-1} N\right)<1$ if and only if $A$ is nonsingular and $A^{-1}$ is nonnegative.

Theorem 2. Let $A=M-N$ be a weak regular splitting of the matrix $A$. Then, $A$ is nonsingular with $A^{-1} \geq O$ if and only if $\rho\left(M^{-1} N\right)<1$.

Definition 2. The spectrum of a matrix $A \in \mathbb{C}$ is defined by $\sigma(A)=\{\lambda \in \mathbb{C}: \operatorname{det}(A-\lambda I)=0\}$. That is, the spectrum of a matrix $A$ is the set of all its eigenvalues.
Definition 3. The spectral radius of a matrix $A$ is denoted by $\rho(A)$ and is defined by $\rho(A)=\max _{\lambda \in \sigma(A)}|\lambda|$.

Definition 4. The (asymptotic) rate of convergence of $A$ is denoted by $R_{\infty}(A)$ and is defined by $R_{\infty}(A)=-\ln \rho(A)$.

Lemma 1 (see [8]). $L\left(a_{1}, \ldots, a_{k} ; \omega\right)=(1-\omega) I+\omega(I-$ $\left.\sum_{i=1}^{k} a_{i} L_{i}\right)^{-1}\left(\sum_{i=1}^{k}\left(1-a_{i}\right) L_{i}+U\right)$, where $L_{i}=D^{-1} E_{i}, \quad i=$ $1,2, \ldots, k$, and $U=D^{-1} F$.

Theorem 3 (see [8]). Let $(0, \ldots, 0 ; 0) \leq\left(a_{1}, \ldots\right.$, $\left.a_{k} ; \omega\right) \leq(1, \ldots, 1 ; 1)$ and $\omega \neq 0$. Let $B_{J}=\sum_{i=1}^{k} L_{i}+U$. Then,

(1) $1-\omega \leq \rho\left(L\left(a_{1}, \ldots, a_{k} ; \omega\right)\right)<1$ if and only if $\rho\left(B_{J}\right)<1$; in this case, we have $\rho\left(L\left(a_{1}, \ldots\right.\right.$, $\left.\left.a_{k} ; \omega\right)\right) \leq 1-\omega+\omega \rho\left(B_{J}\right)$. Furthermore, if $\rho\left(B_{J}\right)=0$, then $\rho\left(L\left(a_{1}, \ldots, a_{k} ; \omega\right)\right)=1-\omega$.

(2) $\rho\left(L\left(a_{1}, \ldots, a_{k} ; \omega\right)\right)=1$ if and only if $\rho\left(B_{J}\right)=1$.

(3) $\rho\left(L\left(a_{1}, \ldots, a_{k} ; \omega\right)\right)>1$ if and only if $\rho\left(B_{J}\right)>1$; in this case, we have $\rho\left(L\left(a_{1}, \ldots, a_{k} ; \omega\right)\right) \leq 1-\omega+\omega \rho\left(B_{J}\right)$.

Theorem 4 (see [9]). Let $A=D-E-E^{T}$ be an $n \times n$ Hermitian positive definite matrix, where $D$ is the Hermitian and positive definite and $E$ is strictly lower part of $D-A$. Then, the eigenvalues $\mu_{i}$, where $i=1,2, \ldots, n$, and the Jacobi matrix $B_{J}=D^{-1}\left(E+E^{T}\right)=I-D^{-1} A\left(\equiv L_{0,1}\right)$ are real and less than 1. Moreover, if $\mu_{m}=\min _{i} \mu_{i}$ and $\mu_{M}=\max _{i} \mu_{i}$ and $\mu_{m} \leq 0 \leq \mu_{M}$, then for the values

$$
\omega \in(0,2),
$$

$$
\omega+\frac{2-\omega}{\mu_{m}}<\gamma<\omega+\frac{2-\omega}{\mu_{M}},
$$

provided that $\operatorname{det}(D-\gamma E) \neq 0$, and the AOR method converges.

Theorem 5 (see [2]). Let $A=D-E_{1}-E_{2}-F$ be an $H$ matrix, then the TOR method converges for $0 \leq a_{1}<\omega<2 /\left(1+\rho\left(B_{J}\right)\right)$ and $0 \leq a_{2}<\omega<2 /\left(1+\rho\left(B_{J}\right)\right)$, where $B_{J}=L_{a_{1}, a_{2}, \omega}=L_{0,0,1}$.

\section{Main Results}

Let $A x=b$ be given with $A$ is any nonsingular square matrix. Then, split $A$ as $A=D-\sum_{i=1}^{k} E_{i}-F$, where $D$ is the diagonal matrix, $E_{i}$ is any strictly lower triangular matrix of $D-A$ for $i=1,2, \ldots, k$, and $F$ is strictly upper triangular matrix of $D-A$. Then, $\omega A=\omega D-\sum_{i=1}^{k} \omega E_{i}-\omega F$.

$$
\begin{aligned}
\omega A x & =\omega b \\
\left(D-\sum_{i=1}^{k} a_{i} E_{i}\right)-\left[(1-\omega) D+\sum_{i=1}^{k}\left(\omega-a_{i}\right) E_{i}+\omega F\right] x & =\omega b
\end{aligned}
$$




$$
\begin{aligned}
\left(D-\sum_{i=1}^{k} a_{i} E_{i}\right) x & =\left[(1-\omega) D+\sum_{i=1}^{k}\left(\omega-a_{i}\right) E_{i}+\omega F\right] x+\omega b \\
& =\left[\left(D-\sum_{i=1}^{k} a_{i} E_{i}\right)-\omega A\right] x+\omega b \\
& =\left(D-\sum_{i=1}^{k} a_{i} E_{i}\right) x+\omega(b-A x) \\
x & =x+\omega\left(D-\sum_{i=1}^{k} a_{i} E_{i}\right)^{-1}(b-A x), \\
x^{(n+1)} & =\tilde{x}^{(n+1)}+\omega\left(D-\sum_{i=1}^{k} a_{i} E_{i}\right)^{-1}\left(b-A \tilde{x}^{(n+1)}\right) .
\end{aligned}
$$

After some steps, one can get the following:

$$
x^{(n+1)}=\left(D-\sum_{i=1}^{k} a_{i} E_{i}\right)^{-1}\left[(1-\omega) D+\sum_{i=1}^{k}\left(\omega-a_{i}\right) E_{i}+\omega F\right] \widetilde{x}^{(n+1)}+\omega\left(D-\sum_{i=1}^{k} a_{i} E_{i}\right)^{-1} b .
$$

Equation (5) is called refiner for the multiparameter overrelaxation (MPOR) method. To get the RMPOR method, first we should substitute the MPOR method into equation (5) or equation (4) and then simplify and rearrange, and the results will be

$$
\begin{aligned}
x^{(n+1)}= & {\left[\left(D-\sum_{i=1}^{k} a_{i} E_{i}\right)^{-1}\left((1-\omega) D+\sum_{i=1}^{k}\left(\omega-a_{i}\right) E_{i}+\omega F\right)\right]^{2} x^{(n)} } \\
& +\omega\left(I+\left(D-\sum_{i=1}^{k} a_{i} E_{i}\right)^{-1}\left((1-\omega) D+\sum_{i=1}^{k}\left(\omega-a_{i}\right) E_{i}+\omega F\right)\right)\left(D-\sum_{i=1}^{k} a_{i} E_{i}\right)^{-1} b .
\end{aligned}
$$

Equation (6) is the refinement of multiparameter overrelaxation (RMPOR) iterative method.

Equation (6) can also be written as

$$
x^{(n+1)}=L_{a_{i}, \omega}^{2} x^{(n)}+\left(I+L_{a_{i}, \omega}\right) C_{a_{i}, \omega}
$$

where $\quad L_{a_{i}, \omega}=\left(D-\sum_{i=1}^{k} a_{i} E_{i}\right)^{-1}\left[(1-\omega) D+\sum_{i=1}^{k}\left(\omega-a_{i}\right)\right.$ $\left.E_{i}+\omega F\right]$ and $C_{a_{i}, \omega}=\omega\left(D-\sum_{i=1}^{k} a_{i} E_{i}\right)^{-1} b$.

For a specific $\omega$ and $a_{i}, i=1,2, \ldots, k$, the RMPOR method reduces to

(i) Refinement of Jacobi (RJ) if $a_{1}=a_{2}=a_{3}=\cdots=$ $a_{k}=0$ and $\omega=1$

(ii) Refinement of Gauss-Seidel (RGS) if $a_{1}=a_{2}=\cdots=$ $a_{k}=1$ and $\omega=1$ (iii) Refinement of successive overrelaxation (RSOR) if $a_{1}=a_{2}=\cdots=a_{k}=\omega$ and any $\omega$

(iv) Refinement of Jacobi overrelaxation (RJOR) if $a_{1}=$ $a_{2}=\cdots=a_{k}=0$ and any $\omega$

(v) Refinement of accelerating overrelaxation (RAOR) if $a_{1}=a_{2}=a_{3}=\cdots=a_{k}=\gamma$ and any $\omega$

(vi) Refinement of two-parameter overrelaxation (RTOR) if $a_{1}, a_{2}$, and $\omega$, and the remaining parameters $a_{3}=a_{4}=\cdots=a_{k}=0$, and so on

\subsection{Convergence of RMPOR Method}

Theorem 6. If $A$ is the SDD matrix, then MPOR is convergent for $0 \leq a_{i} \leq 1$ and $0<\omega<1$. 
Proof.

$$
\begin{aligned}
& L_{a_{i} \omega} x=\lambda x \\
& \left((1-\omega) D+\sum_{i=1}^{k}\left(\omega-a_{i}\right) E_{i}+\omega F\right) x=\lambda\left(D-\sum_{i=1}^{k} a_{i} E_{i}\right) x \\
& \left((1-\omega) D+\left(\omega-a_{1}\right) E_{1}+\left(\omega-a_{2}\right) E_{2}+\cdots+\left(\omega-a_{k}\right) E_{k}+\omega F\right) x=\lambda\left(D-a_{1} E_{1}-a_{2} E_{2}-\cdots-a_{k} E_{k}\right) x \\
& \left((1-\omega) D+\omega\left(E_{1}+E_{2}+\cdots+E_{k}\right)+(\lambda-1)\left(a_{1} E_{1}+a_{2} E_{2}+\cdots+a_{k} E_{k}\right)+\omega F\right) x=\lambda D x \\
& (1-\omega-\lambda) D x+\omega(E+F) x=(1-\lambda)\left(a_{1} E_{1}+a_{2} E_{2}+\cdots+a_{k} E_{k}\right) x \\
& (1-\omega-\lambda) a_{i i} x_{i}-\omega \sum_{j=1, j \neq i}^{n} a_{i j} x_{j}=(1-\lambda) \sum_{i=1}^{k} a_{i} a_{i, j} x_{i} \\
& \left|1-\omega-\lambda\left\|a_{i i}\right\| x_{i}\right|-|\omega| \sum_{j=1, j \neq i}^{n}\left|a_{i j}\right|\left|x_{j}\right| \leq|1-\lambda| \sum_{j=1, j \neq i}^{k}\left|a_{i}\left\|a_{i, j}\right\| x_{i}\right| \\
& \frac{|1-\omega-\lambda|}{|1-\lambda|} \leq \sum_{j=1, j \neq i}^{k} \frac{\left|a_{i}\right|\left|a_{i, j}\right|}{\left|a_{i i}\right|}+\frac{|\omega|}{|1-\lambda|} \sum_{j=1, j \neq i}^{n} \frac{\left|a_{i j}\right|}{\left|a_{i i}\right|} \\
& <\sum_{j=1, j \neq i}^{k} \frac{\left|a_{i}\right|\left|a_{i, j}\right|}{\left|a_{i i}\right|}+\frac{|\omega|}{|1-\lambda|} \\
& \frac{|1-\omega-\lambda|-|\omega|}{|1-\lambda|}<\sum_{j=1, j \neq i}^{k} \frac{\left|a_{i}\right|\left|a_{i, j}\right|}{\left|a_{i i}\right|}<1
\end{aligned}
$$

for $0 \leq a_{i} \leq 1$. Thus,

$$
\frac{|1-\omega-\lambda|-|\omega|}{|1-\lambda|}<1
$$

According to Theorem 3, $\rho\left(L_{a_{i}, \omega}\right)<1$ since A is SDD for which $\rho(B)<1 \quad$ or let $D^{-1} E_{i}=\left(l_{k j}^{(i)}\right), \quad D^{-1} F=\left(u_{k j}\right)$, $l_{k}^{(i)}=\sum_{j=1}^{k-1}\left|l_{k j}^{(i)}\right|$, and $u_{k}=\sum_{j=k+1}^{n}\left|l_{k j}^{\left|u_{k j}\right|}\right|$. If $A$ is strictly diagonally dominant, then we have $\left\|B_{J}\right\|_{\infty}<1$ and $\sum_{i=1}^{k}\left|a_{i}\right| l_{k}^{(i)}<1$, and we obtain

$$
\rho\left(L_{a_{i}, \omega}\right) \leq \max \frac{|1-\omega|+\sum_{i=1}^{k}\left|\omega-a_{i}\right| l_{k}^{(i)}+\omega u_{k}}{1-\sum_{i=1}^{k}\left|a_{i}\right| l_{k}^{(i)}} .
$$

If $\omega \leq 1$, inequality (10) reduces to

$$
\begin{aligned}
\rho\left(L_{a_{i}, \omega}\right) & \leq \max \frac{1-\omega+\sum_{i=1}^{k}\left(\omega-a_{i}\right) l_{k}^{(i)}+\omega u_{k}}{1-\sum_{i=1}^{k} a_{i} l_{k}^{(i)}} \\
& \leq \max \frac{1-\omega+\omega\left\|B_{J}\right\|_{\infty}-\sum_{i=1}^{k} a_{i} l_{k}^{(i)}}{1-\sum_{i=1}^{k} a_{i} l_{k}^{(i)}}<1 .
\end{aligned}
$$

If $\omega>1$, then inequality (10) reduces to

$$
\begin{aligned}
\rho\left(L_{a_{i}, \omega}\right) & \leq \max \frac{\omega-1+\sum_{i=1}^{k}\left(a_{i}-\omega\right) l_{k}^{(i)}+\omega u_{k}}{1-\sum_{i=1}^{k} a_{i} l_{k}^{(i)}} \\
& \leq \max \frac{\omega-1+\omega\left\|B_{J}\right\|_{\infty}-\sum_{i=1}^{k} a_{i} l_{k}^{(i)}}{1-\sum_{i=1}^{k} a_{i} l_{k}^{(i)}}<1 .
\end{aligned}
$$

Hence, the MPOR method is convergent. For $L_{\omega, a}$ having real eigenvalue, the method is also convergent for $1-\omega<\lambda<1-(1 / 2 \omega)$. Hence, the optimal value is $1-(1 / 2 \omega)$.

Theorem 7. If $A$ is symmetric and positive definite (SPD) and $D-\sum_{i=1}^{k} a_{i} E_{i}$ is nonsingular, then the MPOR method is convergent. 
Proof.

$$
\begin{array}{r}
\left(\begin{array}{r}
L_{a_{i}, \omega} x \\
\left.(1-\omega) D+\sum_{i=1}^{k}\left(\omega-a_{i}\right) E_{i}+\omega F\right)
\end{array}\right) \lambda\left(D-\sum_{i=1}^{k} a_{i} E_{i}\right) x \\
x^{T}\left((1-\omega) D+\sum_{i=1}^{k}\left(\omega-a_{i}\right) E_{i}+\omega F\right) x=\lambda x^{T}\left(D-\sum_{i=1}^{k} a_{i} E_{i}\right) x \\
x^{T}\left(D-\omega D+\omega\left(E_{1}+E_{2}+\cdots+E_{k}\right)-\left(a_{1} E_{1}+a_{2} E_{2}+\cdots+a_{k} E_{k}\right)+\omega F\right) x=\lambda x^{T}\left(D-\sum_{i=1}^{k} a_{i} E_{i}\right) x \\
x^{T}\left(D-\sum_{i=1}^{k} a_{i} E_{i}\right) x-\omega x^{T}(D-E-F) x=\lambda x^{T}\left(D-\sum_{i=1}^{k} a_{i} E_{i}\right) x \\
\omega x^{T} A x=(1-\lambda) x^{T}\left(D-\sum_{i=1}^{k} a_{i} E_{i}\right) x .
\end{array}
$$

We now suffice to show that the spectral radius of $G$ satisfies $\rho(G)<1$. Let $\lambda$ be an eigenvalue of $G$, and let $x$ be an eigenvector corresponding to $\lambda$.

$$
\begin{aligned}
G & =\left(D-\sum_{i=1}^{k} a_{i} E_{i}\right)^{-1}\left[(1-\omega) D+\sum_{i=1}^{k}\left(\omega-a_{i}\right) E_{i}+\omega F\right] \\
& =\left(D-\sum_{i=1}^{k} a_{i} E_{i}\right)^{-1}\left[\left(D-\sum_{i=1}^{k} a_{i} E_{i}\right)-\omega A\right] \\
& =I-B^{-1} A
\end{aligned}
$$

where $B=(1 / \omega) D-(1 / \omega) \sum_{i=1}^{k} a_{i} E_{i}$.

From the above, we have

$$
\begin{aligned}
& B^{-1} A=I-G, \\
& A^{-1} B=(I-G)^{-1} .
\end{aligned}
$$

From equation (22), we obtain

$$
\begin{aligned}
G & =I-B^{-1} A \\
B G & =B-A \\
B(I+G) & =2 B-A \\
A^{-1} B(I+G) & =A^{-1}(2 B-A) \\
(I-G)^{-1}(I+G) x & =\mu x \\
(I+G) x & =\mu(I-G) x .
\end{aligned}
$$

If the eigenvalues of $G$ are $\lambda$,

$$
\begin{aligned}
1+\lambda & =\mu(1-\lambda), \\
\mu & =\frac{1+\lambda}{1-\lambda}, \\
\lambda & =\frac{\mu-1}{\mu+1}, \\
|\lambda|^{2} & =\lambda \bar{\lambda}=\frac{|\mu|^{2}+1-\operatorname{Re}(\mu)}{|\mu|^{2}+1+\operatorname{Re}(\mu)} .
\end{aligned}
$$

Thus, we have $\lambda<1$ since $\operatorname{Re}(\mu)>0$. Therefore, the MPOR method is convergent.

Theorem 8. If $A$ is an $M$-matrix and $0 \leq a_{i} \leq \omega<1$, then the MPOR iterative method is convergent.

Proof. In the MPOR iterative method, we have $A=D-$ $\sum_{i=1}^{k} E_{i}-F$ or $A=M-N$ where $M=1 / \omega\left(D-\sum_{i=1}^{k} a_{i} E_{i}\right)$ and $N=\left[(1-\omega / \omega) D+\sum_{i=1}^{k}\left(\omega-a_{i} / \omega\right) E_{i}+F\right]$. From this, we have $A \leq M$. Therefore, $M$ is an M-matrix and as a result $M^{-1} \geq O$. On the other hand, for $0<a_{i}<1$, we have $\rho\left(\sum_{i=1}^{k}\left(a_{i} D^{-1} E_{i}\right)\right)<1$, and therefore,

$$
\begin{aligned}
M^{-1} N & =\left(D-\sum_{i=1}^{k} a_{i} E_{i}\right)^{-1}\left[(1-\omega) D+\sum_{i=1}^{k}\left(\omega-a_{i}\right) E_{i}+\omega F\right] \\
& =\left(I-\sum_{i=1}^{k} a_{i} D^{-1} E_{i}\right)^{-1}\left[(1-\omega) I+\sum_{i=1}^{k}\left(\omega-a_{i}\right) D^{-1} E_{i}+\omega D^{-1} F\right]
\end{aligned}
$$




$$
=\sum_{j=0}^{\infty}\left(\sum_{i}^{k}\left(a_{i} D^{-1} E_{i}\right)\right)^{j}\left[(1-\omega) I+\sum_{i=1}^{k}\left(\omega-a_{i}\right) D^{-1} E_{i}+\omega D^{-1} F\right] \geq 0 .
$$

For $0 \leq a_{i} \leq \omega<1$.

Hence, we conclude that is $A$ a weak regular splitting.

Thus, using Theorem 2 , we conclude that $\rho\left(M^{-1} N\right)<1$. This completes the proof.

Theorem 9. Let $A$ be strictly diagonally dominant and $B_{J}=D^{-1}\left(\sum_{i=1}^{k} E_{i}+F\right)=\sum_{i=1}^{k} L_{i}+U=I-D^{-1} A$. Then, for

$$
0 \leq a_{i} \leq \omega<\frac{2}{1+\left\|B_{J}\right\|_{\infty}}, \quad \omega \neq 0,
$$

the RMPOR method converges.

Proof. Assume $0 \leq a_{i} \leq \omega$ and $L_{a_{i}, \omega}=\left(\left(D-\sum_{i=1}^{k} a_{i} E_{i}\right)^{-1}\right.$ $\left.\left((1-\omega) D+\sum_{i=1}^{k}\left(\omega-a_{i}\right) E_{i}\right)+\omega F\right)^{2}$. It is known that $\left\|B_{J}\right\|_{\infty}<1$ since $A$ is SDD matrix.

Then,

$$
\begin{aligned}
L_{a_{i}, \omega} x & =\lambda x \\
\left(D-\sum_{i=1}^{k} a_{i} E_{i}\right)^{-1}\left((1-\omega) D+\sum_{i=1}^{k}\left(\omega-a_{i}\right) E_{i}+\omega F\right) x & =\lambda^{1 / 2} x \\
\left((1-\omega) D+\sum_{i=1}^{k}\left(\omega-a_{i}\right) E_{i}+\omega F\right) x & =\lambda^{1 / 2}\left(D-\sum_{i=1}^{k} a_{i} E_{i}\right) x \\
\left((1-\omega) I+\sum_{i=1}^{k}\left(\omega-a_{i}\right) L_{i}+\omega U\right) x & =\lambda^{1 / 2}\left(I-\sum_{i=1}^{k} a_{i} L_{i}\right) x \\
\left(\lambda^{1 / 2} \sum_{i=1}^{k} a_{i} L_{i}+\omega \sum_{i=1}^{k} L_{i}-\sum_{i=1}^{k} a_{i} L_{i}+\omega U\right) x & =\left(\lambda^{1 / 2}+\omega-1\right) x \\
\left(\lambda^{1 / 2} \sum_{i=1}^{k} a_{i} L_{i}-\sum_{i=1}^{k} a_{i} L_{i}+\omega B_{J}\right) x & =\left(\lambda^{1 / 2}+\omega-1\right) x
\end{aligned}
$$

Taking norms on both sides, we get

$\left|\lambda^{1 / 2}+\omega-1\right|\|x\|_{\infty} \leq\left(\left|\lambda^{1 / 2}-1\right|\left\|\sum_{i=1}^{k} a_{i} L_{i}\right\|_{\infty}+\omega\left\|B_{J}\right\|_{\infty}\right)\|x\|_{\infty}$.

Thus,

$$
\left|\lambda^{1 / 2}+\omega-1\right| \leq\left|\lambda^{1 / 2}-1\right|\left\|\sum_{i=1}^{k} a_{i} L_{i}\right\|_{\infty}+\omega\left\|B_{J}\right\|_{\infty} .
$$

Using inequalities,

$$
\begin{gathered}
\left|\lambda^{1 / 2}-1\right|-|\omega| \leq\left|\lambda^{1 / 2}-1\right|\left\|\sum_{i=1}^{k} a_{i} L_{i}\right\|_{\infty}+\omega\left\|B_{J}\right\|_{\infty}, \\
\left|\lambda^{1 / 2}-1\right|\left\|\sum_{i=1}^{k} a_{i} L_{i}\right\|_{\infty} \leq \omega\left(1+\left\|B_{J}\right\|_{\infty}\right), \\
0<\left|\lambda^{1 / 2}-1\right|\left(1-\left\|\sum_{i=1}^{k} a_{i} L_{i}\right\|_{\infty}\right)<\omega\left(1+\left\|B_{J}\right\|_{\infty}\right)<2 .
\end{gathered}
$$

From the fact that $\left\|\sum_{i=1}^{k} a_{i} L_{i}\right\|_{\infty}<1$ and $\lambda<1$, $\left|\lambda^{1 / 2}-1\right|<1$. Therefore,

$$
\omega<\frac{2}{1+\left\|B_{J}\right\|_{\infty}} .
$$

Hence, we prove the theorem.

Theorem 10. If $A$ is symmetric and positive definite (SPD) and $\operatorname{det}\left(D-\sum_{i=1}^{k} a_{i} L_{i}\right) \neq 0$, then the refinement of multiparameter overrelaxation (RMPOR) method is convergent for any initial guess $x^{(0)}$.

Proof. Let us begin with

$$
\begin{aligned}
L_{a_{i}, \omega} x & =\lambda x \\
\left((1-\omega) D+\sum_{i=1}^{k}\left(\omega-a_{i}\right) E_{i}+\omega F\right) x & =\lambda\left(D-\sum_{i=1}^{k} a_{i} E_{i}\right) x
\end{aligned}
$$

$$
(\lambda+\omega-1) D x+(1-\lambda) \sum_{i=1}^{k} a_{i} E_{i} x=\omega\left(\sum_{i=1}^{k} E_{i}+F\right) x
$$




$$
(\lambda+\omega-1) I x+(1-\lambda) \sum_{i=1}^{k} a_{i} L_{i} x=\omega\left(\sum_{i=1}^{k} L_{i}+U\right) x .
$$

However, we know that the eigenvalues of the Jacobi matrix $B_{J}$ is $\mu$. Thus,

$$
\begin{aligned}
B_{J} x & =\mu x, \\
D^{-1}\left(\sum_{i=1}^{k} E_{i}+F\right) x & =\mu x, \\
\left(\sum_{i=1}^{k} L_{i}+U\right) x & =\mu x, \\
\omega\left(\sum_{i=1}^{k} L_{i}+U\right) x & =\omega \mu x .
\end{aligned}
$$

Using equations (29) and (31), we get

$$
\begin{aligned}
(\lambda+\omega-1) I x+(1-\lambda) \sum_{i=1}^{k} a_{i} L_{i} x & =\omega \mu x, \\
(\lambda+\omega-1-\omega \mu) x & =(\lambda-1) \sum_{i=1}^{k} a_{i} L_{i} x .
\end{aligned}
$$

From equation (33), we have the following two cases:

(1) If $x^{*} \sum_{i=1}^{k} a_{i} E_{i} x>0$, then $\lambda+\omega-1-\omega \mu / \lambda-1>0$. Again, from this, we have two cases:

(i) $\lambda-1>0$ and $\lambda+\omega-1-\omega \mu>0$. Thus, $\lambda>1$ and $\lambda+\omega-1-\omega \mu>0$ which is impossible according to convergent property.

(ii) $\lambda-1<0$ and $\lambda+\omega-1-\omega \mu<0$. In this case, we get $\lambda<1$ and $\omega<1-\lambda / 1-\mu$.

On the other hand,

(2) If $x^{*} \sum_{i=1}^{k} a_{i} E_{i} x<0$, then $\lambda+\omega-1-\omega \mu / \lambda-1<0$. Again, from this we, have two cases:

(i) $\lambda-1<0$ and $\lambda+\omega-1-\omega \mu>0$. In this case, we have $\lambda<1$ and $\omega<1-\lambda / 1-\mu$.

(ii) $\lambda-1>0$ and $\lambda+\omega-1-\omega \mu<0$ which is impossible.

Thus, we must choose the second possibility, which is $x^{*} \sum_{i=1}^{k} a_{i} E_{i} x<0$.

Therefore, we can conclude that

$$
x^{*}\left(D-\sum_{i=1}^{k} a_{i} E_{i}\right) x>0 \text {. }
$$

Now, let us start to proof the theorem, using equation

$$
\begin{aligned}
& \left((1-\omega) D+\sum_{i=1}^{k}\left(\omega-a_{i}\right) E_{i}+\omega F\right) x=\lambda\left(D-\sum_{i=1}^{k} a_{i} E_{i}\right) x \\
& (\lambda+\omega-1) D x+(1-\lambda) \sum_{i=1}^{k} a_{i} E_{i} x=\omega\left(\sum_{i=1}^{k} E_{i}+F\right) x \\
& (1-\lambda) D x+(\lambda-1) \sum_{i=1}^{k} a_{i} E_{i} x=\omega\left(D-\sum_{i=1}^{k} E_{i}-F\right) x \\
& (1-\lambda)\left(D-\sum_{i=1}^{k} a_{i} E_{i}\right) x=\omega A x \\
& \frac{1-\lambda}{\omega} x^{*}\left(D-\sum_{i=1}^{k} a_{i} E_{i}\right) x=x^{*} A x \text {. }
\end{aligned}
$$

Using equation (34) and SPD property of the matrix $A$, we have $x^{*}\left(D-\sum_{i=1}^{k} a_{i} E_{i}\right)>0$ and $x^{*} A x>0$. Hence, $1-\lambda>0$. Thus,

$$
\lambda<1 \text {. }
$$

Therefore, the MPOR method is convergent.

Now, let us prove the convergence of the RMPOR iterative method.

$$
\begin{aligned}
& L_{a_{i}, \omega}^{2} x=\Lambda x, \\
& L_{a_{i}, \omega} x=\Lambda^{1 / 2} x,
\end{aligned}
$$

where $\Lambda$ is the eigenvalue of $L_{a_{i}, \omega}^{2}$. From equation (38), we have $\lambda=\Lambda^{1 / 2}$ or $\lambda^{2}=\Lambda$.

From this, we can generalize that the RMPOR method is convergent if A is SPD matrix.

Theorem 11. Let $A$ be an M-matrix. If

$$
0 \leq a_{i} \leq \omega<\frac{2}{1+\rho\left(B_{J}\right)}
$$

and then $\rho\left(L_{a_{i}, \omega}^{2}\right)<1$. That is, the RMPOR iterative method is convergent.

Proof. For the case when $\omega \leq 1$, the statement is proved. Now, we consider the case when $\omega>1$. We assume $\rho\left(L_{a_{i}, \omega}\right)>1$ and denote

$$
L_{a_{i}, \omega}=\left(\left(D-\sum_{i=1}^{k} a_{i} E_{i}\right)^{-1}\left((1-\omega) D+\sum_{i=1}^{k}\left(\omega-a_{i}\right) E_{i}+\omega F\right)\right)^{2} .
$$
(27). 
By direct comparision, it gets. Hence, there exists a nonzero vector $x \geq 0$ such that

$$
\begin{aligned}
L_{a_{i}, \omega} x & =\rho\left(L_{a_{i} \omega}\right) x \\
\left(1+\rho\left(L_{a_{i}, \omega}\right)^{1 / 2}-\omega\right) x & =\left\{\sum_{i=1}^{k}\left(\omega-a_{i}+a_{i} \rho\left(L_{a_{i}, \omega}\right)^{1 / 2}\right) L_{i}+U\right\} x \\
& \leq \max \left(\omega+\left(\rho\left(L_{a_{i}, \omega}\right)^{1 / 2}-1\right) a_{i}\right) B_{J} x . \\
\text { Thus, } & \\
1+\rho\left(L_{a_{i}, \omega}\right)^{1 / 2}-\omega & \leq \max \left(\omega+\left(\rho\left(L_{a_{i}, \omega}\right)^{1 / 2}-1\right) a_{i}\right) \rho\left(B_{J}\right) \\
& \leq \omega \rho\left(L_{a_{i}, \omega}\right)^{1 / 2} \rho\left(B_{J}\right),
\end{aligned}
$$

and consequently,

$$
\omega \geq \frac{1+\rho\left(L_{a_{i}, \omega}\right)^{1 / 2}}{1+\rho\left(L_{a_{i}, \omega}\right)^{1 / 2} \rho\left(B_{J}\right)} \geq \frac{2}{1+\rho\left(B_{J}\right)} .
$$

Obviously, it contradicts the hypothesis; therefore, we have $\rho\left(L_{a_{i}, \omega}\right) \leq \rho\left(L_{a_{i}, \omega}\right)<1$.

\subsection{Numerical Examples}

Example 1. Let us solve the system of linear equations of the form $\mathrm{Ax}=b$ using SOR, AOR, TOR, MPOR, and RMPOR iterative methods, where

$$
A=\left(\begin{array}{cccccccccccc}
8 & -1 & 0 & -1 & 0 & 0 & 0 & 1 & 0 & 1 & 0 & 0 \\
-1 & 8 & -1 & 0 & -1 & 0 & 1 & 0 & 1 & 0 & 1 & 0 \\
0 & -1 & 8 & 0 & 0 & -1 & 0 & 1 & 0 & 0 & 0 & 1 \\
-1 & 0 & 0 & 8 & -1 & 0 & 1 & 0 & 0 & 0 & 1 & 0 \\
0 & -1 & 0 & -1 & 8 & -1 & 0 & 1 & 0 & 1 & 0 & 1 \\
0 & 0 & -1 & 0 & -1 & 8 & 0 & 0 & 1 & 0 & 1 & 0 \\
0 & 1 & 0 & 1 & 0 & 0 & 8 & -1 & 0 & -1 & 0 & 0 \\
1 & 0 & 1 & 0 & 1 & 0 & -1 & 8 & -1 & 0 & -1 & 0 \\
0 & 1 & 0 & 0 & 0 & 1 & 0 & -1 & 8 & 0 & 0 & -1 \\
1 & 0 & 0 & 0 & 1 & 0 & -1 & 0 & 0 & 8 & -1 & 0 \\
0 & 1 & 0 & 1 & 0 & 1 & 0 & -1 & 0 & -1 & 8 & -1 \\
0 & 0 & 1 & 0 & 1 & 0 & 0 & 0 & -1 & 0 & -1 & 8
\end{array}\right),
$$

$$
b=\left(\begin{array}{c}
9 \\
-9 \\
21 \\
18 \\
9 \\
13 \\
23 \\
33 \\
-13 \\
-18 \\
7 \\
19
\end{array}\right)
$$

$$
E_{1}=\left(\begin{array}{cccccccccccc}
0 & 0 & 0 & 0 & 0 & 0 & 0 & 0 & 0 & 0 & 0 & 0 \\
0 & 0 & 0 & 0 & 0 & 0 & 0 & 0 & 0 & 0 & 0 & 0 \\
0 & 0 & 0 & 0 & 0 & 0 & 0 & 0 & 0 & 0 & 0 & 0 \\
1 & 0 & 0 & 0 & 0 & 0 & 0 & 0 & 0 & 0 & 0 & 0 \\
0 & 1 & 0 & 0 & 0 & 0 & 0 & 0 & 0 & 0 & 0 & 0 \\
0 & 0 & 1 & 0 & 0 & 0 & 0 & 0 & 0 & 0 & 0 & 0 \\
0 & -1 & 0 & -1 & 0 & 0 & 0 & 0 & 0 & 0 & 0 & 0 \\
-1 & 0 & -1 & 0 & -1 & 0 & 0 & 0 & 0 & 0 & 0 & 0 \\
0 & -1 & 0 & 0 & 0 & -1 & 0 & 0 & 0 & 0 & 0 & 0 \\
0 & 0 & 0 & 0 & -1 & 0 & 1 & 0 & 0 & 0 & 0 & 0 \\
0 & 0 & 0 & -1 & 0 & -1 & 0 & 1 & 0 & 0 & 0 & 0 \\
0 & 0 & 0 & 0 & -1 & 0 & 0 & 0 & 1 & 0 & 0 & 0
\end{array}\right),
$$


TABle 1: Comparison of SOR, AOR, TOR, MPOR, and RMPOR methods for Example 1.

\begin{tabular}{lccc}
\hline Methods & Number of iterations & Spectral radius & Rate of convergence \\
\hline SOR $\left(\omega_{0}=1.1128\right)$ & 8 & 0.2334 & 1.4550 \\
AOR $(\widehat{\omega}=1$ and $\widehat{\gamma}=1.1128)$ & 10 & 0.2556 & 1.3641 \\
TOR & 16 & 0.5691 & 0.5637 \\
MPOR & 17 & 0.5731 & 0.5567 \\
RMPOR & 9 & 0.3284 & 1.1135 \\
\hline
\end{tabular}

TABle 2: Comparison of SOR, AOR, TOR, MPOR, and RMPOR methods for Example 2.

\begin{tabular}{lccc}
\hline Methods & Number of iterations & Spectral radius & Rate of convergence \\
\hline SOR $\left(\omega_{0}=1.01\right)$ & 6 & 0.1243 & 2.0851 \\
AOR $(\widehat{\omega}=1.01$ and $\hat{\gamma}=0.9)$ & 5 & 0.1364 & 1.9922 \\
TOR & 8 & 0.3143 & 1.1574 \\
MPOR & 8 & 0.3138 & 1.1590 \\
RMPOR & 4 & 0.0985 & 2.3177 \\
\hline
\end{tabular}

$$
E_{3}=\left(\begin{array}{cccccccccccc}
0 & 0 & 0 & 0 & 0 & 0 & 0 & 0 & 0 & 0 & 0 & 0 \\
1 & 0 & 0 & 0 & 0 & 0 & 0 & 0 & 0 & 0 & 0 & 0 \\
0 & 1 & 0 & 0 & 0 & 0 & 0 & 0 & 0 & 0 & 0 & 0 \\
0 & 0 & 0 & 0 & 0 & 0 & 0 & 0 & 0 & 0 & 0 & 0 \\
0 & 0 & 0 & 1 & 0 & 0 & 0 & 0 & 0 & 0 & 0 & 0 \\
0 & 0 & 0 & 0 & 1 & 0 & 0 & 0 & 0 & 0 & 0 & 0 \\
0 & 0 & 0 & 0 & 0 & 0 & 0 & 0 & 0 & 0 & 0 & 0 \\
0 & 0 & 0 & 0 & 0 & 0 & 1 & 0 & 0 & 0 & 0 & 0 \\
0 & 0 & 0 & 0 & 0 & 0 & 0 & 1 & 0 & 0 & 0 & 0 \\
0 & 0 & 0 & 0 & 0 & 0 & 0 & 0 & 0 & 0 & 0 & 0 \\
0 & 0 & 0 & 0 & 0 & 0 & 0 & 0 & 0 & 1 & 0 & 0 \\
0 & 0 & 0 & 0 & 0 & 0 & 0 & 0 & 0 & 0 & 1 & 0
\end{array}\right),
$$

with $a_{1}=0.5, a_{2}=0.6, a_{3}=0.4$, and $\omega=0.9 . \quad E=E_{1}+$ $E_{2}=E_{1}+E_{3}+E_{4}$. To use the MPOR and RMPOR method, we used the splitting $E=E_{1}+E_{3}+E_{4}$, and to apply the TOR method, we used the splitting $E=E_{1}+E_{2}$. The exact solution is $\bar{x}=[1 ;-1 ; 2 ; 2 ; 1 ; 2 ; 3 ; 4 ;-1 ;-2 ; 1 ; 2]$. The spectral radius of $L_{a_{i}, \omega}$ is $\rho\left(L_{a_{i}, \omega}\right)=0.5731$ and $\rho\left(L_{a_{i}, \omega}^{2}\right)=0.3284$ which means that RMPOR is faster than the MPOR iterative method.

From Table 1, the RMPOR method converges to the exact solution faster than the MPOR method. The spectral radius is also smaller than the MPOR method. Similarly, rate of convergence of the RMPOR is larger than that of MPOR method. Hence, the proposed is better than MPOR method.

Example 2 (see [10]). Consider the system of linear equations:

$$
\left\{\begin{array}{l}
6 x_{1}+2 x_{2}+2 x_{3}=5 \\
2 x_{1}+8 x_{2}+2 x_{3}=6 \\
2 x_{1}+2 x_{2}+10 x_{3}=7
\end{array}\right.
$$

The coefficient matrix is both SDD and SPD matrix. To solve the system with tolerance 0.0001 by using SOR, AOR, TOR, MPOR, and RMPOR iterative methods would be shown as follows:

Solution is as follows:

$$
\begin{aligned}
A & =\left(\begin{array}{lll}
6 & 2 & 2 \\
2 & 8 & 2 \\
2 & 2 & 10
\end{array}\right), \\
b & =\left(\begin{array}{l}
5 \\
6 \\
7
\end{array}\right), \\
E_{1} & =\left(\begin{array}{ccc}
0 & 0 & 0 \\
-2 & 0 & 0 \\
0 & 0 & 0
\end{array}\right), \\
E_{2} & =\left(\begin{array}{ccc}
0 & 0 & 0 \\
0 & 0 & 0 \\
-2 & -2 & 0
\end{array}\right), \\
E_{3} & =\left(\begin{array}{ccc}
0 & 0 & 0 \\
0 & 0 & 0 \\
-2 & 0 & 0
\end{array}\right), \\
E_{4} & =\left(\begin{array}{lll}
0 & 0 & 0 \\
0 & 0 & 0 \\
0 & -2 & 0
\end{array}\right),
\end{aligned}
$$


with $a_{1}=0.4, a_{2}=0.5, a_{3}=0.6$, and $\omega=0.9$. We also used the splitting of $E$ as $E=E_{1}+E_{2}$ for the TOR method and $E=E_{1}+E_{3}+E_{4}$ for MPOR and RMPOR iterative methods.

From Table 2, the number of iteration of RMPOR is smaller than that of the MPOR method. The spectral radius of the RMPOR method is smaller than the spectral radius of the MPOR method. Similarly, rate of convergence of RMPOR is larger than that of the MPOR method. Hence, the proposed method is better than the MPOR method.

\section{Conclusion}

Iterative methods are very essential to solve large and sparse linear system of equations which arise from the discretization of PDE and ODE problems. The iterative methods can be found by splitting the coefficient matrix. There are many techniques to split the matrix $A$. One of them is multiparameter splitting technique which is discussed in this paper. The method obtained by this technique is efficient to solve system of linear equations whose coefficient matrices are SDD, SPD, and M-matrices, and then the RMPOR iterative method is better than the MPOR iterative method.

\section{Data Availability}

No data were used to support this study.

\section{Conflicts of Interest}

The authors declare that there are no conflicts of interest regarding the publication of this paper.

\section{Authors' Contributions}

All authors have equally contributed to this work. All authors read and approved the final manuscript.

\section{References}

[1] Y.-Z. Song and S. Dai, "Multi-parameters overrelaxation (MPOR) method for solving linear systems," International Journal of Computer Mathematics, vol. 59, no. 1-2, pp. 77-90, 1995.

[2] J. Kuang and J. Ji, "A survey of AOR and TOR methods," Journal of Computational and Applied Mathematics, vol. 24, no. 1-2, pp. 3-12, 1988.

[3] D.-W. Chang, "Convergence analysis of the parallel multisplitting TOR method," Journal of Computational and Applied Mathematics, vol. 72, no. 1, pp. 169-177, 1996.

[4] M. Neumann and R. J. Plemmons, "Convergence of parallel multisplitting iterative methods for M-matrices," Linear Algebra and Its Applications, vol. 88-89, pp. 559-573, 1987.

[5] L.-T. Zhang, T.-Z. Huang, S.-H. Cheng, T.-X. Gu, and Y.-P. Wang, "A note on parallel multisplitting TOR method for H-matrices," International Journal of Computer Mathematics, vol. 88, no. 3, pp. 501-507, 2011.

[6] E. Tohidi and M. Khorsand Zak, "A new matrix approach for solving second-order linear matrix partial differential equations," Mediterranean Journal of Mathematics, vol. 13, no. 3, pp. 1353-1376, 2015.
[7] Z. Asgari, F. Toutounian, E. Babolian, and E. Tohidi, "An extended block Golub-Kahan algorithm for large algebraic and differential matrix Riccati equations," Computers \& Mathematics with Applications, vol. 79, no. 8, pp. 2447-2457, 2020.

[8] W. Li and Z.-Y. You, "The multi-parameters overrelaxation method," Journal of Computational Mathematics, vol. 16, no. 4, pp. 367-374, 1998.

[9] N. Gaïtanos, A. Hadjidimos, and A. Yeyios, "Optimal accelerated overrelaxation (AOR) method for system with positive definite coefficient matrix," SIAM Journal on $\mathrm{Nu}$ merical Analysis, vol. 20, no. 4, pp. 774-783, 1983.

[10] T. K. Enyew, G. Awgichew, E. Haile, and G. D. Abie, "Secondrefinement of Gauss-Seidel iterative method for solving linear system of equations," Ethiopian Journal of Science and Technology, vol. 13, no. 1, pp. 1-15, 2020. 